Biol. Proced. Online 2004;6(1): 220-225.

doi:10.1251/bpo92

\title{
Screening of Compounds Toxicity against Human Monocytic cell line-THP- 1 by Flow Cytometry
}

\author{
Neora Pick ${ }^{1}$, Scott Cameron' ${ }^{1}$, Dorit Arad ${ }^{1}$ and Yossef Av-Gay ${ }^{*}$ \\ University of British Columbia, Department of Medicine, Division of Infectious Diseases. Vancouver, BC, \\ Canada, V5Z 3J5.
}

*To whom correspondence should be addressed: Yossef Av-Gay, University of British Columbia, Department of Medicine, Division of Infectious Diseases. 2733 Heather Street, D450 HP East, Vancouver, BC, Canada, V5Z 3J5. Phone: 604-875-4588; Email: yossi@interchange.ubc.ca

Submitted: February 25, 2004; Revised: September 17, 2004; Accepted: September 19, 2004; Published: October 1, 2004.

Indexing terms: Flow Cytometry; Propidium; Lethal Dose 50.

Abbreviations: FACS, Fluorescence Activated Cell Sorting; PI, propidium iodide; $\mathrm{LD}_{50}$, lethal dose in which $50 \%$ of the cells die.

\begin{abstract}
The worldwide rapid increase in bacterial resistance to numerous antibiotics requires on-going development of new drugs to enter the market. As the development of new antibiotics is lengthy and costly, early monitoring of compound's toxicity is essential in the development of novel agents. Our interest is in a rapid, simple, high throughput screening method to assess cytotoxicity induced by potential agents. Some intracellular pathogens, such as Mycobacterium tuberculosis primary site of infection is human alveolar macrophages. Thus, evaluation of candidate drugs for macrophage toxicity is crucial. Protocols for high throughput drug toxicity screening of macrophages using flow cytometry are lacking in the literature. For this application we modified a preexisting technique, propidium iodide (PI) exclusion staining and utilized it for rapid toxicity tests. Samples were prepared in 96 well plates and analyzed by flow cytometry, which allowed for rapid, inexpensive and precise assessment of compound's toxicity associated with cell death.
\end{abstract}

\section{INTRODUCTION}

Drug development is a long and costly process. The attritionadjusted cost of research to develop and bring to market a prescription drug in the US has skyrocketed to $\$ 802$ million (1).

Only 1:5000 of the compounds starting the process (starting point defined as lead compounds identification) end up being a drug distributed to the market (2). The Time frame from synthesis to FDA approval of drug and distribution to the public varies between 10-15 years. Cutting development and regulatory review times by $25 \%$ will lower total costs by $\$ 129$ million (3).
The global spread of bacterial resistance to numerous antibiotics requires on going development of new drugs.

Tuberculosis (TB) is the most common infectious disease known to mankind. Incidence of TB is 8 million people per year; prevalence is 1.86 billion people, with case fatality rate of $23 \%$ worldwide (4). Treatment induces many side effects and is long (6-9 months). As a result-multi drug resistant TB spreads. A survey in 72 countries suggested that the multi-drug-resistant (MDR) TB problem is more wide spread than previously thought and is likely worsening (5). Since the organism that causes TB, $M$. tuberculosis is an intracellular pathogen, and its first target is

(C) 2004 by the author(s). This paper is Open Access and is published in Biological Procedures Online under license from the author(s). Copying, printing, redistribution and storage permitted. Journal C 1997-2004 Biological Procedures Online. 
alveolar macrophages, we tested drug candidates on THP-1 cells, which are monocytic cell line that can be differentiated in vitro to macrophages for toxicity. THP-1 are widely used for in vitro infection of $M$. tuberculosis and other intracellular pathogen like Listeria monocytogenes (6) and Legeionella (7). Protocols for high throughput analysis of drug toxicity on THP-1 cells are not well established in the literature.

Fluorescence-Activated Cell Sorting (FACS), also known as Flow Cytometry is an effective method that measures and separates properties of cells in flow. Cells in suspension flow through an illuminated cell where they scatter laser beamed light and emit fluorescence. The fluorescence data is collected, and analyzed according to the cells properties, such as granulation, fluorochrome binding, size etc. This method allows detection of fluorescent dye staining, which is coupled to cell size and granularity determinations. Flow cytometry instruments enable numerous samples to be run in batch automation for screening various potential drugs and doses with little extra effort.

In this report we present a technique for determining compounds toxicity on THP-1 cells. This method allows checking many compounds simultaneously, and is therefore useful for screening processes. This technique is a modification of the propidium iodide exclusion staining for drug toxicity determination: Propidium iodine (PI) is a DNA intercalating dye that is excluded by cells that have their plasma membrane integrity preserved (including live or early apoptotic cells), but it enters and stains DNA in cells that have damaged membranes, and these are considered dead cells (8).

A new family of Bromo-tyrosine compounds active against Gram Positive bacteria including TB and MRSA (9) were examined using the FACS technique described below. We describe the application for EXEG 1706 which was chosen as a representative of 36 initial compounds. We found this method to be a short, efficient, and inexpensive way to precisely evaluate toxicity, as LD50 values were easily obtained for our compounds of interest.

\section{MATERIALS AND METHODS}

\section{Growth of THP-1 cells}

Monocytic cell line THP-1 (ATCC 202, Rockville, MA) was cultured in RPMI 1640 (Hyclone, Logan, UT) supplemented with 5\% Fetal calf serum (FCS), (Hyclone, Logan, UT), and $2 \mathrm{mM} \mathrm{L-}$ glutamine (StemCell Technologies, Vancouver, BC). Cells were seeded at a density of $3 \times 10^{5}$ per well in a 96 well plate, and incubated with the desired drug concentration in triplicates, to a total of $200 \mu \mathrm{l}$, at $37^{\circ} \mathrm{C}$ in a humidified atmosphere of $5 \% \mathrm{CO}_{2}$ for $24 \mathrm{~h}$. We used two negative controls for the tests: 1; THP-1 cells in drug free culture media. 2; THP-1 cells treated for 24 hours with the maximum concentration of the drug's solvent used in the experiments (4\% methanol). As a positive control for a compound with apparent activity, THP-1 cells were treated for 6 hours with $5 \% \mathrm{H}_{2} \mathrm{O}_{2}$, and subsequently stained with PI. This induced approximately $50 \%$ cell death in our experiments (data not shown). To confirm that there is a strong correlation between positive PI staining and cell death we sorted PI negative cells using FACS caliber cytometer and found that $95 \%$ of the sorted cells were negative for Trypan blue staining.

\section{Flow cytometers}

A Beckman Coulter EPICS XL MCL (Beckman Coulter, Fullerton, CA, USA) equipped with an 32 sample automated carousel was used for acquisition of samples. The FL3 gate was sensitive to light at $610-625 \mathrm{~nm}$, which was ideal for detecting emmisions of PI when bound to DNA, which is excited at $535 \mathrm{~nm}$, and emits at $615 \mathrm{~nm}$. EXPO32 ADC (Applied Cytometry Systems, V 1.1 build 207) software was used for subsequent analysis. Sorting of PI positive cells was performed on a BD FACS Vantage SE Turbo sort cell sorter (BD biosciences, Mississauga, ON, Canada), for confirmation of cell permeability using Trypan blue staining. Other models of flow cytometers would be useful for this application provided they have a laser capable of exciting PI, and a filter capable of detecting it.

The only staining used was PI, and the control was initially used as the background, which were THP-1 cells with no additional drug, or THP-1 cells with the solvent at maximum concentration.

\section{Drugs used}

EXEG 1706, a Bromo-tyrosine derivative with molecular weight of $778.33 \mathrm{~g} / \mathrm{mol}$ dissolved in 95\% HPLC grade methanol was used (9).

\section{Creation of $\mathrm{PI}+$ gate}

Untreated fresh THP-1 cells after 2-3 passages were stained with PI by the protocol outlined, and used as a negative control for PI staining. After 24 hours of incubation of THP-1 cells with the compound, a gate was drawn selecting a small portion (usually 5 $7 \%$ ) of cells staining with FL3 intensities over $10^{1}$, using a histogram of the FL3 channel. This gate was then adjusted using the cell death positive control, or a sample treated with the highest concentration of drug, which resulted in a histogram containing a large secondary peak of PI positive cells around $10^{2}$. The gate was adjusted to include this second peak. Alternatively, as an additional positive control, THP-1 cells were treated for 6 hrs with $\mathrm{H}_{2} \mathrm{O}_{2}$ in RPMI. As a negative control-methanol 4\% was used since it was the maximal concentration of the solvent used in the experiment of the tested compound.

\section{Creation of forward (FS) / side scatter (SS) dead cells gate}

Utilizing the untreated negative control for PI staining as outlined above, a large dead cell gate is drawn which is to the top and left of the healthy cell population. This gate is then adjusted with the positive control for PI staining, to make sure that it includes the majority of cells which have shifts in their forward/side scatter characteristics.

During analysis of cytotoxicity, it is important to correlate changes in forward and side scatter characteristics with positive PI staining, since positively stained PI cells should become 
smaller and more granular. This is achieved by comparing the number of dead cells gated via the FS/SS dead cell gate with the number of PI positive cells. The PI gate is used as the gold standard in this analysis, though the FS/SS dead cell gate should correlate well with the PI gate, to within $\pm 10 \%$.

\section{RESULTS AND DISCUSSION}

EXEG 1706 was the selected compound to be presented. The assay was performed on 7 lead compounds out of 36 initial compounds tested. The basis for selecting EXEG 1706 was a newly designed family of compounds that were found to be very active against TB and MRSA using disc assays and Minimal Inhibitory Concentration (MIC) assays. Toxicity tests are usually done in animal models, but in order to reduce the cost of drug development, preliminary in vitro toxicity assays are required to ensure that the least toxic compounds are pursued.
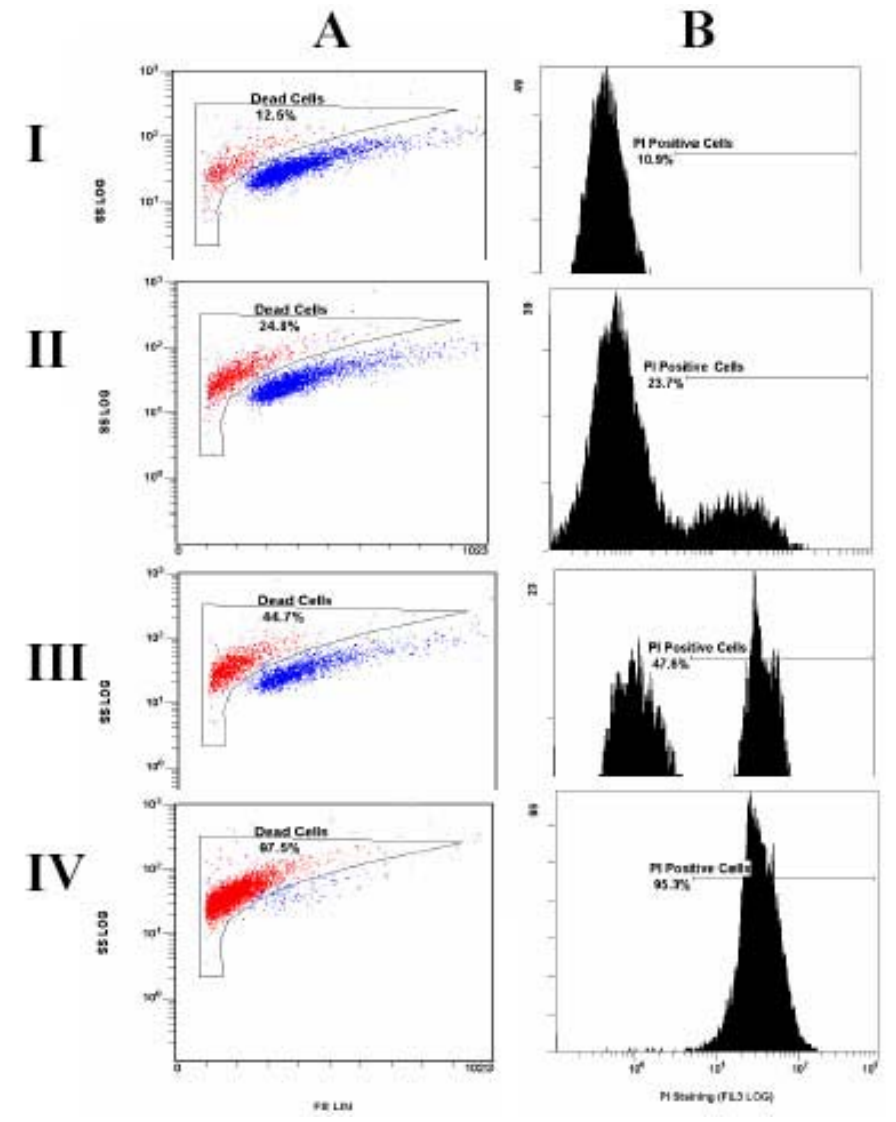

Fig. 1: Effect of Compound treatment on THP-1 cell membrane permeability as measured by PI exclusion staining. Linear forward scatter vs Log side scatter dot plots (A) histograms of PI stain intensity detected on the FL3 channel (B) of THP-1 cells treated for 24 hours with a lead compound being tested for drug toxicity. (I) Healthy THP-1 cells in the log phase of growth, treated with drug carrier ( $4 \%$ methanol in culture medium), and stained with PI as outlined. $10.9 \%$ are dead as determined by the PI gate. This correlates well with the indicated dead cell gate based on changes in forward and side scatter. (II) THP-1 cells treated with $50 \mu \mathrm{g} / \mathrm{ml}$ of the test compound, $23.7 \%$ are PI positive. (III) THP-1 cells treated with $100 \mu \mathrm{g} / \mathrm{ml}$ of the test compound, $47.6 \%$ are PI positive. (IV) THP-1 cells treated with $200 \mu \mathrm{g} / \mathrm{ml}$ of the test compound, $95.3 \%$ are PI positive $=$ dead cells.
Figure 1 shows the effect of EXEG 1706 treatment on THP-1 cell membrane permeability by PI exclusion staining. The toxicity effect was measured both by linear forward scatter (X axis) versus $\log$ side scatter ( $\mathrm{Y}$ axis). Our gating is based on the difference in florescence of target cells-undifferentiated THP-1 (monocytes) with and without the addition of the tested compound at different concentrations. As expected, for the two negative controls used, (THP-1 cells in drug free culture media, and THP-1 cells treated for 24 hours with 4\% methanol-the maximum concentration of the drug's solvent used in the experiments) no significant toxicity was observed. As presented in Figure 2 the toxicity effect as measured by the death rate of THP-1 cells was dose dependent.

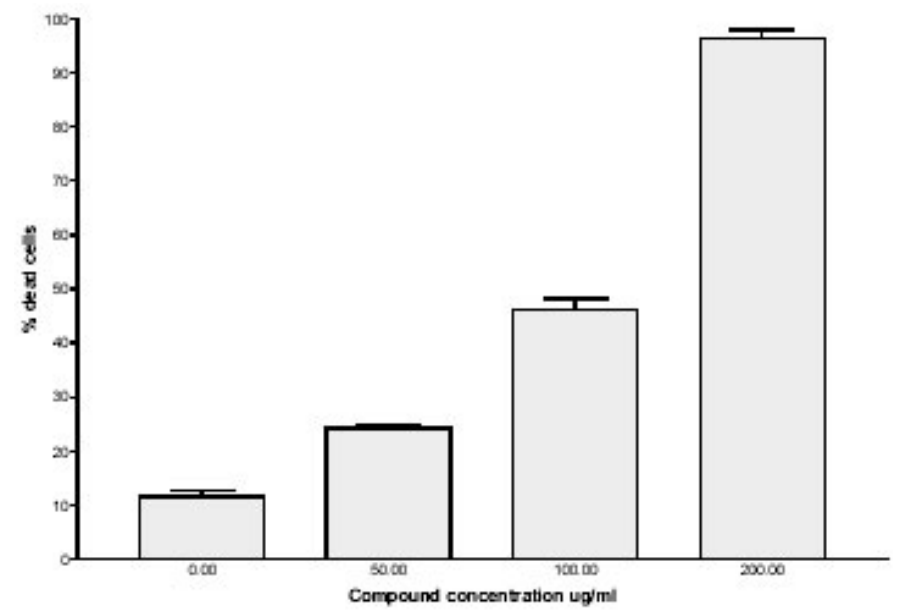

Fig. 2: Histogram representation of the effect of the EXEG 1706 tested on undifferentiated THP-1 cells, as measured by flow cytometry.

This method allowed us to determine the calculated $\mathrm{LD}_{50}$. For this compound $\mathrm{LD}_{50}$ is approximately $100 \mu \mathrm{g} / \mathrm{ml}$. Since the MIC of this compound is $2-16 \mu \mathrm{g} / \mathrm{ml}$ (9), we could conclude that the drug is relatively not toxic to the monocytic cell line tested. Assessment of different $\mathrm{LD}_{50}$ to THP-1 cells from various compounds provide a determination of the tested compounds safe zone, (the non-harmful dose). This technique allows us to compare the toxicity within different compounds of the same family, a valuable tool for high throughout screening of various drugs from a combinatorial chemistry effort. We used actinomycin D as an external reference to validate our method. As seen in Figure 3, the toxicity effect is linear between the range of $1 \mathrm{ng}$ and $10 \mathrm{ug} / \mathrm{ml}$. The established $\mathrm{LD}_{50}$ for actinomycin $\mathrm{D}$ in THP-1 is about $120 \mathrm{ng} / \mathrm{ml}$ and is comparable to that described in the literature for various cell lines.

PI staining and subsequent FACs analysis is used for different applications, however a clear protocol for high throughout toxicity could not be found in the literature. Our use of THP-1 samples prepared in round bottomed 96 well plates allow quantitative analysis of toxicity, and minimize the amount of cells, drug and staining reagents required for toxicity assessment. The use of a flow cytometer with the ability to read directly from 
a 96 well plate would further increase the throughput of this system.

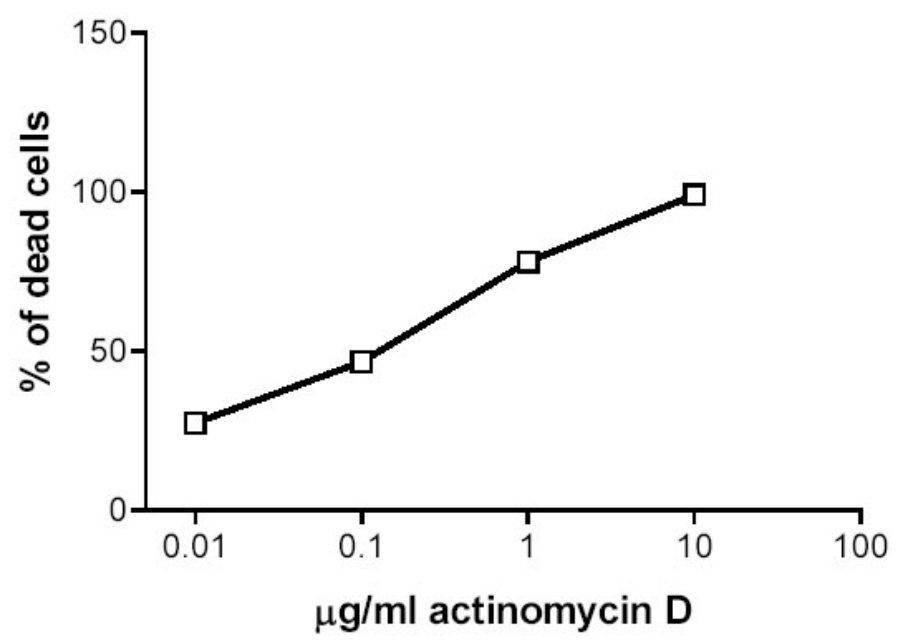

Fig. 3: The effect of actinomycin D on undifferentiated THP-1 cells, as measured by flow cytometry.

The observed shift in SSC that resulted from our drug treatment would indicate an increase in cellular granularity, consistent with apoptosis being the route of cell death induced by EXEG 1706. Further investigation using more specific methods such as Annexin $\mathrm{V}$ and 7AAD staining would be required to specify the mechanism of cell death (10). 7AAD is comparable to PI staining in detecting membrane permeability: It is used for the exclusion of nonviable cells as well (11). Annexin V staining is capable of detecting apoptosis, thus revealing the type of cell death induced by the compound of interest. The combination of Annexin V and PI has been used to discriminate early apoptotic cells from late apoptotic and necrotic ones, based on the translocation of phosphatidyl serine from the inner to the outer layer of the plasma membrane of early apoptotic cells stained by annexin $\mathrm{V}$. $(10,12-15)$. While this alternative method would provide a more mechanistic explanation of toxicity, this technique is significantly more expensive due to reagent cost: $\$ 2.50 /$ sample (if the reagents are prepared and standardized by you). Common kits can cost as much as $\$ 4.28$ / sample, which is extremely expensive compared to $\$ 0.05 /$ sample with the PI method as outlined. Therefore, we suggest that the mechanism of cellular toxicity of a given compound is not required at the preliminary stage of highthroughput screening, and is better left to later studies of the few promising agents identified during the screening process.

As previously mentioned, we screened 36 initial compounds for disc sensitivity and MIC, Only the most promising seven compounds were chosen for further testing with the discussed assay. Different compounds within the same family showed different toxicity range on the same THP-1 cells (data not shown). We have determined that the toxicity of each compound is dose dependent; however, we cannot conclude that this pattern of toxicity would be the same in other compounds, and this needs to be checked individually for each tested compound.

\section{CONCLUSIONS}

This data and protocol indicate that PI exclusion staining in a 96 well plate, with subsequent flow cytometric analysis is a methodology well suited for high-throughput screening. Early toxicity assessment of cells in suspension can be determined for candidate-drugs treatment using a simple, low cost method that can provide rapid results.

\section{ACKNOWLEDGMENTS}

We thank Zakaria Hmama and Ryan Hung for critically reviewing this manuscript. Funding for this project was provided by eXegenics pharmaceuticals Inc.

\section{REFERENCES}

1. Tufts Center for the Study of Drug Development. Impact report 4(5) Sept 2002.

2. Tufts Center for the Study of Drug Development. Impact report 5(3) May 2003.

3. Alliance pharmaceutical corp: phases of product development. Drug development and approval process; p. 13. www.allp.com/drug_dev.htm

4. Dye C, Scheele S, Dolin P, Pathania V, Raviglione MC. Consensus statement. Global burden of tuberculosis: estimated incidence, prevalence, and mortality by Country. WHO Global Surveillance and Monitoring Project, JAMA 1999; 282(7):677-686.

5. WHO/IUATLD. Global project on anti-tuberculosis drug resistance in the world: Second report. World Health Organization/CDS/2000.278.

6. Carryn S, Van Bambeke F, Mingeot-Leclercq MP, Tulkens PM. Comparative intracellular (THP-1 Macrophage) and extracellular activities of B-lactams, Azithromycin, Gentamicin and Fluoroquinolones against Listeria monocytogenes at clinically relevant concentrations. Anti Agent Chemo 2002; 46(7):2095-2103.

7. Takemura H, Hiroyuki Yamamato H, Kunishima H, Ikejima $\mathrm{H}$, Takashi $\mathrm{H}$ et al. Evaluation of a human monocytic cell line THP-1 model for assay of the intracellular activities of antimicrobial agents against Legionella pneumophila. J Antimicrob Chemother 2000; 46(4):589-594.

8. Darzynkiewicz Z, Bruno S, Del Bino G, Gorczyca W, Hotz MA, Lassota P, Traganos F. Features of Apoptotic Cells Measured by Flow Cytometry. Cytometry 1992; 13: 795- 808.

9. Pick N, Good RL, Arad D, Av-Gay Y. EXEG 1706: A novel antimicrobial agent against Methicillin-resistant Staphylococcus aureus. Abs- F-2147 (pp. 269) ICAAC, Chicago 2003.

10. Vermes I, Haanen C, Steffens-Nakken H, Reutelingsperger C. A novel assay for apoptosis. Flow cytometric detection of phosphatidylserine expression on early apoptotic cells using 
fluorescein labeled Annexin V. J Immunol Methods 1995; 184:39-51.

11. Schmid I, Krall WJ, Uittenbogaart CH, Braun J, Giorgi JV. Dead cell discrimination with 7-amino-actinomycin D in combination with dual color immunofluorescence in single laser flow cytometry. Cytometry 1992; 13(2):204-208.

12. Darzynkiewicz,Z, Juan G, Li X, Gorezvca W, Murakami T, Traganos F. Cytometry in cell necrobiology: analysis of apoptosis and accidental cell death (necrosis). Cytometry 1997; 27(1):1-20.

13. Sherwood SW, Schimke RT. Cell cycle analysis of apoptosis using flow cytometry. Methods Cell Biol 1995; 46:77-97.

14. Telford WG, King LE, Fraker PJ. Comparative evaluation of several DNA binding dyes in the detection of apoptosisassociation chromatin degradation by flow cytometry. Cytometry 1992; 13(2):137-143.

15. Bertho AL, Santiago MA, Coutinho SG. Flow cytometry in the study of cell death. Mem Inst Oswaldo Cruz 2000; 95(3):429-433. 


\section{PROTOCOLS}

\section{Protocol for in vitro toxicity of novel potential antibiotic compounds on THP-1 cells}

\section{Reagents}

- FACs buffer: Fresh 3\% Fetal calf serum (Gibco, Cat no $16000-044)$ stored at $-20^{\circ} \mathrm{C}$, in Phosphate buffer saline (PBS) $\mathrm{x} 1(\mathrm{pH}=$ 7.4).

- PI Staining buffer: $10 \mathrm{mM}$ stock solution in $\mathrm{H}_{2} \mathrm{O}$ (Stored at $4^{\circ} \mathrm{C}$ in the dark, since it is light sensitive).

- THP-1 monocytic cell line (ATCC 202, Rockville, MA).

- Cell culture medium: RPMI (Minimal tissue culture media) 1640 (SIGMA) with the addition of 5\% Fetal calf serum $+1 \%$ Glutamine.

- Flat bottom 96 wells Elisa plate (Corning 3595) and U bottom Elisa plate (Falcon 3077 microtest U-bottom).

- $5 \% \mathrm{H}_{2} \mathrm{O}_{2}$ for a positive control.

Note: Wear gloves, since Propidium Iodide may be carcinogenic and mutagenic

- Suspend $3 \times 10^{5}$ /well THP-1 human monocytes in 50-100 $\mu$ l tissue culture medium RPMI) in a flat bottom Elisa plate (Corning 3595). To minimize well to well cross-contamination during staining, space filled wells so that each will be surrounded by empty wells on all sides.

- Add compound to be tested at the desired concentrations, to reach a final volume of $200 \mu \mathrm{l}$ per well.

- Incubate the filled plate, covered, for 24 hours at $37^{\circ} \mathrm{C}$ in $5 \% \mathrm{CO}_{2}$.

- Resuspend the cells by pipeting up and down 2 times, then transfer the content of each well to a 96 wells U-shaped bottom plate (Falcon 353077 microtest U-bottom).

- Centrifuge at $800 \mathrm{xg}$ for 1 minute.

- Flick liquid sharply from plate to sink.

- Add $100 \mu \mathrm{l}$ of freshly prepared FACs buffer to each well containing the macrophages.

- Centrifuge again in $800 \mathrm{xg}$ for 1 minute. Repeat the washing and centrifuging twice.

- Check for pellet.

- Dilute Propidium iodide (PI) $10 \mathrm{mM}$ stock - 1:100 in FACs buffer.

- Add $100 \mu \mathrm{l} 0.1 \mathrm{mM}$ Propidium iodide to each well.

- Refrigerate at $4^{\circ} \mathrm{C}$ for 20 minutes to let PI penetrate the dead cells

- Centrifuge at $800 \mathrm{xg}$ for 1 minute. Flick Sharply

- Wash with $100 \mu \mathrm{FACs}$ buffer, spin at 800xg for one more minute, and flick. Repeat this step twice.

- After flicking the third time-add $200 \mu \mathrm{FACs}$ buffer again to each well, and resuspend the cells in this buffer.

- Transfer the $200 \mu \mathrm{l}$ of cells into 5-ml plastic tubes containing $300 \mu \mathrm{l} \mathrm{FACs} \mathrm{buffer,} \mathrm{previously} \mathrm{aliquoted.} \mathrm{Cover} \mathrm{with} \mathrm{silver} \mathrm{foil.}$

- Read in Flow cytometry machine - 10,000 events/ sample. Histogram gates were established on the FL3 channel on the basis of the cell's incorporation of PI. Three parameters were evaluated; \% positive PI cells and cellular forward/side scatter, which represent cell size and granularity, respectively.

- Analyze data using dedicated software such as Beckman Coulter supplied software (EXPO 32 ADC V1.2). 